\title{
Reducing computational time via order reduction of a class of reaction-diffusion systems
}

\author{
F. López-Caamal, M. R. García*, R. H. Middleton
}

\begin{abstract}
In this paper, we consider a class of reactiondiffusion PDEs. For this class, a suitable state transformation allows conversion to a heat equation together with a lower order PDE set. By giving an explicit solution to the heat equation we are able to obtain a complete solution to the original PDE. By focusing on the computational load, we give a comparison of the pure numerical, analytical/numerical, analytical/approximated, and approximated methods of solving the PDE. In some examples, we note an almost order of magnitude improvement in computational load.
\end{abstract}

\section{INTRODUCTION}

Partial Differential Equations (PDEs) appear as a natural way to describe a plethora of systems given the behaviour that only these equations can reproduce. Their application spectrum, in a biological context, is as rich as their dynamical behaviour, ranging from population dynamics to metabolic pathways [1]-[3].

In general, the numerical solution of these PDEs involves the integration of a large set of ordinary differential equations (ODEs). Moreover, the approaches based on spatial discretization, such as Finite Element Method, are usually computationally involved and present a number of disadvantages as some essential control theoretic properties such as controllability or observability may be lost by the discretization scheme or the degree of refinement [4].

Due to these disadvantages, new methods based on spectral decomposition techniques, have emerged. This approach avails of the spatial differential operator structure along with the Galerkin method to approximate the system by a lowdimensional set of ODEs [5].

In this work, we focus on a class of continuous time reaction-diffusion systems, whose stoichiometric matrix allows for a linear transformation that represents the system as an interconnection of a linear and nonlinear PDEs. The analytical solution of the former PDE in addition to the numerical resolution of the Galerkin method, provide a novel approach to solve the original nonlinear PDE. Also we present a matrix notation for the ODEs that arises from the Galerkin method, which has not been reported in the literature previously, to the best of our knowledge.

The authors are with the Hamilton Institute, National University of Ireland Maynooth, Co. Kildare, Ireland. Tel: + 353 (0)1 7086100.

This work was supported by Science Foundation Ireland via grant 07/IN.1/I1838 and through the National Biophotonics and Imaging Platform, Ireland, and funded by the Irish Government's Programme for Research in Third Level Institutions, Cycle 4, Irelands EU Structural Funds Programmes 2007-2013.

* Author for correspondence: Miriam.Garcia@nuim.ie
A comparison of the computational load for the pure numerical, analytical/numerical, analytical/approximated, and approximated methods is presented.

\section{SYSTEM DESCRIPTION}

A reaction network is composed of a group of species, whose chemical interaction is represented by

$$
\sum_{i=1}^{n} a_{i j} S_{i} \stackrel{v_{j}}{\longrightarrow} \sum_{i=1}^{n} b_{i j} S_{i} .
$$

Here $S_{i}$ is the ith reactant or product for the $j t h$ reaction. The symbols $a_{i j}$ and $b_{i j}$ denote the stoichiometric coefficients of the corresponding species. We will focus on biological systems that can be idealized as adiabatic and isothermal. This allows us to fully determine the state of the network exclusively from the species' concentration. A compact notation for this kind of system is given by

$$
\dot{\mathbf{c}}=\mathbf{N v}(\mathbf{c}) \text {. }
$$

Here, $\mathbf{c}(t): \mathbb{R}_{+} \rightarrow \mathbb{R}^{n}$ comprises the trajectories of the species concentrations in (1). The rate at which the reactants are becoming products compose the vector $\mathbf{v}(\mathbf{c}(t)): \mathbb{R}_{+} \rightarrow$ $\mathbb{R}^{m}$. Despite the turn-over, these reaction rates are nonlinear functions of the state $\mathbf{c}(t)$, which can be modelled by wellknown principles such as the Mass Action Law, MichaelisMenten kinetics, and Hill kinetics. The link from reaction rates to the actual concentration change is the stoichiometric matrix $\mathbf{N} \in \mathbb{R}^{n \times m}$, whose $i j t h$ element is defined as

$$
N_{i j}=b_{i j}-a_{i j} .
$$

We further assume that the reaction rate vector is split into nonlinear, linear and constant functions, i.e.,

$$
\mathbf{v}(\mathbf{c}(t))=\left(\begin{array}{lll}
\mathbf{v}_{\mathbf{n l}}{ }^{T} & \mathbf{v}_{\mathbf{l}}{ }^{T} & \mathbf{v}_{\mathbf{0}}{ }^{T}
\end{array}\right)^{T}: \mathbb{R}_{+} \rightarrow \mathbb{R}^{m_{1}+m_{2}+m_{3}} .
$$

The linearity in the state of the elements in $\mathbf{v}_{\mathbf{l}}(\mathbf{c})$ can be written explicitly as

$$
\mathbf{v}_{\mathbf{l}}(\mathbf{c})=\Gamma \mathbf{c},
$$

where $\boldsymbol{\Gamma} \in \mathbb{R}^{m_{2} \times n}$.

The ordering in (3) induces the following partition in the stoichiometric matrix

$$
\mathbf{N}=\left(\begin{array}{lll}
\mathbf{N}_{\mathbf{n l}} & \mathbf{N}_{\mathbf{l}} & \mathbf{N}_{\mathbf{0}}
\end{array}\right) \in \mathbb{R}^{n \times\left(m_{1}+m_{2}+m_{3}\right)} .
$$

Although the model in (2) determines the temporal profile of the system, it fails to reproduce some behaviours in which the parameter uncertainty, spatial behaviour [1], [2], 
and/or geometric constrains are of interest. All this effects can be modelled by PDEs, which, in contrast to the ODEs, are functions which include derivatives w.r.t more than one variable.

Here we will focus on the spatio-temporal behaviour of the reaction network, compelled by the diffusion of species in only one spatial direction, denoted as $x \in \Omega \subset \mathbb{R}$. This effect will be modelled by Fick's laws [6], from which we obtain, (see [1], for example)

$$
\frac{\partial}{\partial t} \mathbf{c}(t, x)=\mathbf{D} \nabla_{x}^{2} \mathbf{c}(t, x)+\mathbf{N} \mathbf{v}(\mathbf{c}(t, x)) .
$$

The initial and spatial restrictions are defined by initial and boundary conditions:

$$
\begin{aligned}
\mathbf{c}(0, x)= & \mathbf{c}_{0}(x) \forall x \in \Omega \subset \mathbb{R} \\
m(t, \partial \Omega)= & p(t, \partial \Omega) \mathbf{c}(t, \partial \Omega)+\left.q(t, \partial \Omega) \frac{\partial \mathbf{c}(t, x)}{\partial \mathbf{n}}\right|_{x=\partial \Omega} \\
& \forall t \in \mathbb{R}_{+} .
\end{aligned}
$$

The last equation represent the Robin boundary conditions. When $q(t, \partial \Omega)=0$ this expression becomes Dirichlet boundary conditions. Alternatively, by setting $p(t, \partial \Omega)=0$ we have Neumann boundary conditions.

Unless otherwise stated, we will consider systems that satisfy the following properties on the diffusion and reaction mechanisms.

A1) The matrix D in (6) is a scalar, $d$, times the identity

A2) All the reactions in (1) have at most two reactants, and are modelled using Mass-Action kinetics.

In order to deal with the general system in (6), we introduce a change of variables that expresses the system in a form such that the linear and nonlinear terms appear explicitly.

Proposition 2.1: Consider a system of the form (6). Assume the existence of an equilibrium point $\overline{\mathbf{c}} \in \mathbb{R}_{+}^{n}$, s.t $\mathbf{N v}(\overline{\mathbf{c}})=\mathbf{0}$, and that the assumptions A1 and A2 are satisfied, then the dynamics of the system in the coordinates $\mathbf{e}(t, x)=\mathbf{c}(t, x)-\overline{\mathbf{c}}$ are given by

$$
\frac{\partial}{\partial t} \mathbf{e}(t, x)=d \nabla_{x}^{2} \mathbf{e}(t, x)+\mathbf{A e}(t, x)+\mathbf{N}_{\mathbf{n l}} \mathbf{g}(\mathbf{e}(t, x)) .
$$

Here $\quad \mathbf{A}=\mathbf{N}_{\mathbf{n l}} \mathbf{J}_{\mathbf{n l}}(\overline{\mathbf{c}})+\mathbf{N}_{\mathbf{l}} \mathbf{J}_{\mathbf{l}} . \quad$ Moreover $\mathbf{J}_{\mathbf{n l}}=\left.\frac{\partial}{\partial \mathbf{c}} \mathbf{v}_{\mathbf{n l}}\right|_{\mathbf{c}=\overline{\mathbf{c}}}, \mathbf{J}_{\mathbf{l}}=\frac{\partial}{\partial \mathbf{c}} \mathbf{v}_{\mathbf{l}}$, and $\mathbf{g}(\mathbf{e}(t, x))=\mathbf{v}_{\mathbf{n l}}(\mathbf{e}(t, x))$. The proof follows from the Taylor expansion of (6) around $\mathbf{c}=\overline{\mathbf{c}}$. Note that the expression in (9) is exact.

\section{REDUCED ORDER MODELS (ROM)}

Solutions for system (5) with boundary conditions (7) can be found in Hilbert spaces equipped with an inner product and norm of the form

$$
\begin{aligned}
\langle f(x), g(x)\rangle_{\Omega} & =\int_{\Omega} f(x) g(x) \mathrm{d} x, \\
\|f(x)\|_{\Omega} & =\langle f(x), f(x)\rangle_{\Omega}^{1 / 2},
\end{aligned}
$$

where $f(x)$ and $g(x)$ are given functions over the spatial domain $\Omega$. We will consider a set of functions $\phi_{i}(x)$ as the solution of the following integral eigenvalue problem

$$
\int_{\Omega} R(x, \xi) \phi_{i}(\xi) \mathrm{d} \xi=\lambda_{i} \phi_{i}(x) .
$$

Depending on the nature of the kernel $R(x, \xi)$, two methods are usually considered: $i$ ) Proper Orthogonal Decomposition (POD), where $R(x, \xi)$ is a two point correlation matrix constructed from empirical data [7] and ii) Laplacian Spectral Decomposition (LSD), in which $R(x, \xi)$ is the Green function associated with the Laplacian operator [8].

In this work, we will focus on the LSD approach, hence we will consider a special set of functions $\phi_{i}(x)$ invariant w.r.t. the Laplacian operator; that is to say, the solution to the eigenvalue problem in (10) satisfies

$$
\nabla_{x}^{2} \phi_{i}(x)=\lambda_{i} \phi_{i}(x)
$$

where $\lambda_{i}$ corresponds to the eigenvalue associated with $\phi_{i}(x)$. Moreover, we will require the eigenfunctions $\phi_{i}(x)$ to satisfy the boundary conditions in (8) and we will consider that the eigenfunctions are orthonormal w.r.t each other, i.e.,

$$
\left\langle\phi_{i}(x), \phi_{j}(x)\right\rangle=\delta_{i j}
$$

A complete set of functions $\phi_{i}(x)$, will act as a global basis for the Hilbert space to which these functions belong. Let $\mathbf{c}(t, x)$ be an element of such Hilbert space, then it can be expanded as [8]

$$
\mathbf{c}(t, x)=\sum_{i=1}^{\infty} \mathbf{w}_{\mathbf{i}}(t) \phi_{i}(x),
$$

where $\mathbf{w}_{\mathbf{i}}(t): \mathbb{R}_{+} \rightarrow \mathbb{R}^{n}$ collects the so-called modes of $\phi_{i}(x)$.

Due to the dissipative nature of the Laplacian, a truncation of the infinite sum in (13) will provide a good approximation [9]. That is to say

$$
\mathbf{c}(t, x) \approx \sum_{i=1}^{p} \mathbf{w}_{\mathbf{i}}(t) \phi_{i}(x):=\mathbf{W}^{\mathbf{T}}(t) \phi(x),
$$

here $\mathbf{W}^{\mathbf{T}}(t): \mathbb{R}_{+} \rightarrow \mathbb{R}^{n \times p}$ and $\phi(x): \Omega \rightarrow \mathbb{R}^{p}$.

We also note that $\mathbf{W}^{\mathbf{T}}(t)$ satisfies the following relationship, given the orthonormality of the entries in $\phi(x)$,

$$
\begin{aligned}
\langle\mathbf{c}(t, x), \phi(x)\rangle & =\left\langle\mathbf{W}^{\mathbf{T}}(t) \phi(x), \phi(x)\right\rangle \\
\int_{\Omega} \mathbf{c}(t, x) \phi^{\mathbf{T}}(x) \mathrm{d} x & =\int_{\Omega} \mathbf{W}^{\mathbf{T}}(t) \phi(x) \phi^{\boldsymbol{T}}(x) \mathrm{d} x \\
\int_{\Omega} \mathbf{c}(t, x) \boldsymbol{\phi}^{\mathbf{T}}(x) \mathrm{d} x & =\mathbf{W}^{\mathbf{T}}(t) .
\end{aligned}
$$

In the following, for the sake of readability, we will frequently suppress the dependence on space and time in $\mathbf{W}(t)$ and $\phi(x)$.

The next theorem, which relates the interaction of the vectorisation operation vec (o) and the Kroneker product $\otimes$, will be useful in the following.

Theorem 3.1:

$$
\operatorname{vec}(\mathbf{A X B})=\left(\mathbf{B}^{\mathbf{T}} \otimes \mathbf{A}\right) \operatorname{vec}(\mathbf{X}) .
$$

The properties of the operations above as well as the proof of the theorem can be found in [10].

The following proposition shows how to express (6) as an associated first order ODE of dimension $p n$. Here we do not assume that the diffusion is the same for all the species nor a prescribed form of the nonlinear terms in $\mathbf{v}_{\mathbf{n l}}(\mathbf{c})$. 
Proposition 3.1: Consider the PDE defined in (6), with $\mathbf{N}$ and $\mathbf{v}(\mathbf{c})$ as defined in (5) and (3), respectively. The weights $\mathbf{W}(t)$ for which

$$
\mathbf{c}(t, x) \approx \mathbf{W}^{\mathbf{T}}(t) \phi(x),
$$

satisfy the ODE set

$$
\begin{aligned}
\frac{\mathrm{d}}{\mathrm{d} t} \operatorname{vec}\left(\mathbf{W}^{\mathbf{T}}\right)= & \mathbf{A}_{\mathbf{c}} \operatorname{vec}\left(\mathbf{W}^{\mathbf{T}}\right)+\mathbf{B}_{\mathbf{0}} \operatorname{vec}\left(\mathbf{W}_{\mathbf{0}}^{\mathbf{T}}\right) \\
& +\mathbf{B}_{\mathbf{n l}} \int_{\Omega} \operatorname{vec}\left(\mathbf{v}_{\mathbf{n l}}\left(\mathbf{W}^{\mathbf{T}} \boldsymbol{\phi}\right) \boldsymbol{\phi}^{\mathbf{T}}\right) \mathrm{d} x
\end{aligned}
$$

where

$$
\begin{aligned}
\mathbf{A}_{\mathbf{c}} & =(\mathbf{\Lambda} \otimes \mathbf{D})+\left(\mathbf{I}_{\mathbf{p}} \otimes \mathbf{N}_{\mathbf{l}} \boldsymbol{\Gamma}\right) \\
\mathbf{B}_{\mathbf{0}} & =\mathbf{I}_{\mathbf{p}} \otimes \mathbf{N}_{\mathbf{0}} \\
\mathbf{B}_{\mathbf{n l}} & =\mathbf{I}_{\mathbf{p}} \otimes \mathbf{N}_{\mathbf{n l}}, \\
\mathbf{W}_{\mathbf{0}}^{\mathbf{T}} & =\left(\mathbf{v}_{0} \mathbf{0}_{m_{3} \times(p-1)}\right),
\end{aligned}
$$

where $\boldsymbol{\Lambda} \in \mathbb{R}^{p \times p}$ is a diagonal matrix, whose $i i-t h$ entry is $\lambda_{i}$.

Proof: Firstly we note, that expressing (6) as (14), postmultiplying the l.h.s by $\phi^{T}$ and integrating over the spatial domain yield

$$
\int_{\Omega} \frac{\partial}{\partial t} \mathbf{W}^{\mathbf{T}} \boldsymbol{\phi} \phi^{\boldsymbol{T}} \mathrm{d} x=\frac{\mathrm{d}}{\mathrm{d} t} \mathbf{W}^{\mathbf{T}} .
$$

Accordingly, the same operation is applied to the r.h.s of (6), considering the partition in (3) and (5), which leads to

$$
\begin{array}{r}
\frac{\mathrm{d}}{\mathrm{d} t} \mathbf{W}^{\mathbf{T}}= \\
\mathbf{D W}^{\mathbf{T}} \boldsymbol{\Lambda}+\mathbf{N}_{\mathbf{l}} \boldsymbol{\Gamma} \mathbf{W}^{\mathbf{T}}+\mathbf{N}_{\mathbf{0}}\left(\mathbf{v}_{\mathbf{z}} \mathbf{0}\right) \\
+\mathbf{N}_{\mathbf{n l}} \int_{\Omega} \mathbf{v}_{\mathbf{n l}}\left(\mathbf{W}^{\mathbf{T}} \boldsymbol{\phi}\right) \boldsymbol{\phi}^{\mathbf{T}} \mathrm{d} x .
\end{array}
$$

Here we have used the properties in (12) and (11). Vectorising the expression above, yields

$$
\begin{aligned}
\frac{\mathrm{d}}{\mathrm{d} t} \operatorname{vec}\left(\mathbf{W}^{\mathbf{T}}\right)= & \mathbf{A}_{\mathbf{c}} \operatorname{vec}\left(\mathbf{W}^{\mathbf{T}}\right)+\mathbf{B}_{\mathbf{0}} \operatorname{vec}\left(\mathbf{W}_{\mathbf{0}}^{\mathbf{T}}\right) \\
& +\mathbf{B}_{\mathbf{n l}} \int_{\Omega} \operatorname{vec}\left(\mathbf{v}_{\mathbf{n l}}\left(\mathbf{W}^{\mathbf{T}} \boldsymbol{\phi}\right) \boldsymbol{\phi}^{\mathbf{T}}\right) \mathrm{d} x,
\end{aligned}
$$

where the matrices $\mathbf{A}_{\mathbf{c}}, \mathbf{B}_{\mathbf{0}}$ and $\mathbf{B}_{\mathbf{n l}}$ have been defined in (17).

Consider the special case in which $\mathbf{v}_{\mathbf{n l}}: \Omega \times \mathbb{R}_{+} \rightarrow \mathbb{R}^{m_{1}}$ is a vector whose $i t h$ component is a quadratic form, i.e.,

$$
\mathbf{v}_{\mathbf{n l}}(\mathbf{c})=\left(\begin{array}{c}
\mathbf{c}^{\mathbf{T}} \mathbf{P}_{1} \mathbf{c} \\
\vdots \\
c^{\mathbf{T}} \mathbf{P}_{\mathbf{m}_{1}} \mathbf{c}
\end{array}\right) .
$$

Postmultiplication of the expression above by $\phi^{T}$ yields

$$
\begin{gathered}
\mathbf{v}_{\mathrm{nl}}\left(\boldsymbol{W}^{T} \phi\right) \phi^{T}= \\
\left(\begin{array}{c}
\phi^{T} \mathbf{W P}_{1} \mathbf{W}^{\mathbf{T}} \\
\vdots \\
\phi^{T} \mathbf{W} \mathbf{P}_{\mathbf{m}_{1}} \mathbf{W}^{\mathbf{T}}
\end{array}\right) \phi \phi^{T}= \\
\left(\mathbf{I}_{\mathbf{m}_{1}} \otimes \phi^{T}\right)\left(\begin{array}{c}
\mathbf{W} \mathbf{P}_{1} \mathbf{W}^{\mathbf{T}} \\
\vdots \\
\mathbf{W P}_{\mathbf{m}_{1}} \mathbf{W}^{\mathbf{T}}
\end{array}\right) \phi \phi^{T}= \\
\left(\mathbf{I}_{\mathbf{m}_{1}} \otimes \phi^{T}\right)\left(\mathbf{I}_{\mathbf{m}_{1}} \otimes \mathbf{W}\right) \mathbf{P} \mathbf{W}^{\mathbf{T}} \phi \phi^{T} .
\end{gathered}
$$

Here $\mathbf{P} \in \mathbb{R}^{m_{1} n \times n}$ is a matrix obtained by stacking the matrices $\mathbf{P}_{i}$ one below another. Applying vec (o) to the expression above, we have

$$
\begin{array}{r}
\operatorname{vec}\left(\mathbf{v}_{\mathbf{n l}}\left(\boldsymbol{W}^{\boldsymbol{T}} \boldsymbol{\phi}\right) \boldsymbol{\phi}^{\boldsymbol{T}}\right) \\
\left(\boldsymbol{\phi} \phi^{\boldsymbol{T}} \otimes \mathbf{I}_{\mathbf{m}_{1}} \otimes \phi^{\boldsymbol{T}}\right)\left(\mathbf{W} \otimes \mathbf{I}_{\mathbf{m}_{1}} \otimes \mathbf{W}\right) \operatorname{vec}(\mathbf{P}),
\end{array}
$$

from which we note an explicit separation of the spatial and temporal dependencies.

\section{FURTHER REDUCTION VIA ANALYTICAL SOLUTION}

In this section, we present a linear transformation that allows us to write the system in (9) as an interconnection of a linear and a nonlinear system. The analytical solution for the former will be found for an infinite spatial domain, hence the need for a numerical solution will only be necessary for the nonlinear system. We further assume that the spatial domain in which the nonlinear PDE is being simulated is large enough so as to be able to assume it infinite.

Before stating the main result, we present two Lemmas.

Lemma 4.1: Consider a reaction-diffusion system of the form

$$
\frac{\partial}{\partial t} \mathbf{e}=d \nabla_{x}^{2} \mathbf{e}+\mathbf{A e} .
$$

The change of variables $\mathbf{e}=\exp (\mathbf{A} t) \boldsymbol{\gamma}$ in (19) gives

$$
\frac{\partial}{\partial t} \gamma=d \nabla_{x}^{2} \gamma
$$

The proof follows form the substitution of variables. Furthermore, we note that each element of (20) is a homogeneous heat equation.

In the case of the Cauchy problem the use of a Green function will convey a solution. That is to say, the solutions to the entries in (20) are given by [11], [12]

$$
\gamma_{i}(t, x)=\int_{-\infty}^{\infty} g_{i}(\xi) G(t, x, \xi) \mathrm{d} \xi,
$$

where $g_{i}(x)$ is the initial concentration of $\gamma_{i}(t, x)$ and $G(t, x, \xi)$ is a function defined as

$$
G(t, x, \xi)=\frac{1}{2 \sqrt{\pi d t}} \exp \left(-\frac{(x-\xi)^{2}}{4 d t}\right) .
$$

Although this problem is similar to that of our interest, it does not to include the nonlinearities of the system in (9). The next Lemma shows how a basis of the orthogonal complement of $\mathbf{N}_{\mathbf{n l}}$ 's column space, denoted as $\mathbf{Q}$, can induce a linear transformation. The new coordinates will lead to a heat equation, and hence an explicit formula for the solution can be found by means of (21). Let $r=\operatorname{colrank}\left(\mathbf{N}_{\mathbf{n l}}\right)$. By the Rank-Nulity Theorem, $\mathbf{Q} \in \mathbb{R}^{(n-r) \times n}$.

Lemma 4.2: Consider the system in (9). If

$$
\mathrm{QN}_{\mathrm{l}} \mathrm{J}_{1}=\Theta \mathbf{Q},
$$

where $\Theta \in \mathbb{R}^{(n-r) \times(n-r)}$, then the change of variables $\exp (\boldsymbol{\Theta} t) \boldsymbol{\eta}=\mathbf{Q e}$ transforms (9) into an associated PDE set of order $n-r$, composed of homogeneous heat equations

$$
\frac{\partial}{\partial t} \boldsymbol{\eta}=d \nabla_{x}^{2} \boldsymbol{\eta}
$$


Proof: Premultiplying (9) by $\mathbf{Q}$ yields

$$
\frac{\partial}{\partial t} \mathbf{Q e}=d \nabla_{x}^{2} \mathbf{Q e}+\mathbf{Q A e} .
$$

If (22) holds, then

$$
\frac{\partial}{\partial t} \mathbf{Q e}=d \nabla_{x}^{2} \mathbf{Q e}+\mathbf{\Theta Q} \mathbf{e}
$$

Finally, by Lemma 4.1, the change of variables $\exp (\boldsymbol{\Theta} t) \boldsymbol{\eta}=$ Qe leads to the expression

$$
\frac{\partial}{\partial t} \boldsymbol{\eta}=d \nabla_{x}^{2} \boldsymbol{\eta}
$$

as desired.

Remark 4.1: The conditions under which Property (22) holds, can be determined by computing

$$
\Theta=\mathbf{Q N}_{\mathbf{l}} \mathbf{J}_{1} \mathbf{Q}^{+},
$$

and substituting it in (22).

Note that Lemma 4.2 suggest a linear transformation of the form

$$
\left(\begin{array}{l}
\mathbf{y} \\
\mathbf{z}
\end{array}\right)=\left(\begin{array}{c}
\mathbf{Q} \\
\mathbf{M}
\end{array}\right) \mathbf{e}
$$

where $\mathbf{Q} \in \mathbb{R}^{(n-r) \times n}$ and $\mathbf{M} \in \mathbb{R}^{r \times n}$ is a matrix comprised of $r$ linearly independent rows of $\mathbf{N}_{\mathbf{n l}}{ }^{T}$. The dynamics in these coordinates are

$$
\begin{aligned}
\frac{\partial}{\partial t} \mathbf{y} & =d \nabla_{x}^{2} \mathbf{y}+\mathbf{\Theta y} \\
\frac{\partial}{\partial t} \mathbf{z} & =d \nabla_{x}^{2} \mathbf{M e}+\mathbf{M A e}+\mathbf{M N}_{\mathbf{n l}} \mathbf{g}(\mathbf{e})
\end{aligned}
$$

By Lemma 4.2, a closed form expression for $\mathbf{y}(t, x)$ can be found. With the inverse transformation

$$
\mathbf{e}=\left(\begin{array}{ll}
\mathbf{Q}^{+} & \mathbf{M}^{+}
\end{array}\right)\left(\begin{array}{l}
\mathbf{y} \\
\mathbf{z}
\end{array}\right),
$$

we can express the PDE for $\mathbf{z}$ as

$$
\begin{aligned}
\frac{\partial}{\partial t} \mathbf{z}= & d \nabla_{x}^{2} \mathbf{z}+\mathbf{M A M}^{+} \mathbf{z}+\mathbf{M A Q}^{+} \mathbf{y}+ \\
& +\mathbf{M N}_{\mathbf{n l}} \mathbf{g}(\mathbf{y}, \mathbf{z}) .
\end{aligned}
$$

As we see from Equation (25a) and Lemma 4.1 we have available the analytical solution for $\mathbf{y}(t, x)$. Hence it is only necessary to approximate the solution for $\mathbf{z}(t, x)$, in order to reconstruct the full state $\mathbf{c}(t, x)$. Towards this end, the following Proposition shows how the PDE for $\mathbf{z}(t, x)$ can be rewritten as a ROM, by means of the LSD.

Proposition 4.1: Consider the PDE for $\mathbf{z}(t, x)$ as shown in (26). Then $\mathbf{z}(t, x)$ can approximated as

$$
\mathbf{z}(t, x) \approx \mathbf{W}_{\mathbf{z}}^{\mathbf{T}}(t) \phi(x) .
$$

Here $\mathbf{W}_{\mathbf{z}}^{\mathbf{T}}$ satisfies

$$
\begin{aligned}
& \frac{\mathrm{d}}{\mathrm{d} t} \operatorname{vec}\left(\mathbf{W}_{\mathbf{z}}^{\mathbf{T}}\right)=\tilde{\mathbf{A}}_{\mathbf{z}} \operatorname{vec}\left(\mathbf{W}_{\mathbf{z}}^{\mathbf{T}}\right)+\tilde{\mathbf{B}}_{\mathbf{y}} \operatorname{vec}\left(\mathbf{W}_{\mathbf{y}}^{\mathbf{T}}\right) \\
& +\tilde{\mathbf{B}}_{\mathbf{g}} \operatorname{vec}\left(\mathbf{W}_{\mathbf{g}}^{\mathbf{T}}\right), \\
& \mathbf{W}_{\mathbf{g}}^{\mathbf{T}}=\int_{\Omega} \mathbf{g}\left(\mathbf{W}_{\mathbf{y}}^{\mathbf{T}} \phi, \mathbf{W}_{\mathbf{z}}^{\mathbf{T}} \phi\right) \phi^{T} \mathrm{~d} x,
\end{aligned}
$$

and

$$
\begin{aligned}
& \tilde{\mathbf{A}}_{\mathbf{z}}=d\left(\mathbf{\Lambda}^{\mathbf{T}} \otimes \mathbf{I}_{\mathbf{r}}\right)+\left(\mathbf{I}_{\mathbf{p}} \otimes \mathbf{M A M} \mathbf{M A}^{+}\right) \\
& \tilde{\mathbf{B}}_{\mathbf{y}}=\left(\mathbf{I}_{\mathbf{p}} \otimes \mathbf{M A \mathbf { Q } ^ { + }}\right) \\
& \tilde{\mathbf{B}}_{\mathbf{g}}=\left(\mathbf{I}_{\mathbf{p}} \otimes \mathbf{M} \mathbf{N}_{\mathbf{n l}}\right) .
\end{aligned}
$$

Proof: First we note, that postmultiplication of the 1.h.s in (26) by $\phi^{T}$ and integration over the spatial domain yield

$$
\int_{\Omega} \frac{\partial}{\partial t} \mathbf{W}_{\mathbf{z}}^{\mathbf{T}} \phi \phi^{\boldsymbol{T}} \mathrm{d} x=\frac{\mathrm{d}}{\mathrm{d} t} \mathbf{W}_{\mathbf{z}}^{\mathbf{T}} .
$$

Accordingly, the same operation applied to the r.h.s of (26) leads to

$$
\begin{aligned}
\frac{\mathrm{d}}{\mathrm{d} t} \mathbf{W}_{\mathbf{z}}^{\mathbf{T}}= & \quad \mathbf{W}_{\mathbf{z}}^{\mathbf{T}} \boldsymbol{\Lambda}+\mathbf{M A M}^{+} \mathbf{W}_{\mathbf{z}}^{\mathbf{T}}+ \\
& +\mathbf{M A Q}^{+} \mathbf{W}_{\mathbf{y}}^{\mathbf{T}}+\mathbf{N}_{\mathbf{n l}} \mathbf{W}_{\mathbf{g}}^{\mathbf{T}},
\end{aligned}
$$

where the properties in (12) and (11), along with the definition in (28), have been used. We note that $\frac{\mathrm{d}}{\mathrm{d} t} \mathbf{W}_{\mathbf{z}}^{\mathrm{T}}$ is a matrix in $\mathbb{R}^{r \times p}$. Applying vec (o) to the foregoing equation and Theorem 3.1, yield

$$
\begin{aligned}
& \frac{\mathrm{d}}{\mathrm{d} t} \operatorname{vec}\left(\mathbf{W}_{\mathbf{z}}^{\mathbf{T}}\right)=d \operatorname{vec}\left(\mathbf{W}_{\mathbf{z}}^{\mathbf{T}} \boldsymbol{\Lambda}\right)+\operatorname{vec}\left(\mathbf{M} \mathbf{A} \mathbf{M}^{+} \mathbf{W}_{\mathbf{z}}^{\mathbf{T}}\right) \\
& +\operatorname{vec}\left(\mathbf{M A Q}^{+} \mathbf{W}_{\mathbf{y}}^{\mathbf{T}}+\mathbf{M N}_{\mathbf{n l}} \mathbf{W}_{\mathbf{g}}^{\mathbf{T}}\right) \\
& \frac{\mathrm{d}}{\mathrm{d} t} \operatorname{vec}\left(\mathbf{W}_{\mathbf{z}}^{\mathbf{T}}\right)=\tilde{\mathbf{A}}_{\mathbf{z}} \operatorname{vec}\left(\mathbf{W}_{\mathbf{z}}^{\mathbf{T}}\right)+\tilde{\mathbf{B}}_{\mathbf{y}} \operatorname{vec}\left(\mathbf{W}_{\mathbf{y}}^{\mathbf{T}}\right) \\
& +\tilde{\mathbf{B}}_{\mathbf{g}} \operatorname{vec}\left(\mathbf{W}_{\mathbf{g}}^{\mathbf{T}}\right)
\end{aligned}
$$

which is Equation (27) when the definitions in (29) are taken into account.

\section{CAse Study}

\section{A. Problem definition}

We exemplify the use of the procedures above, by means of the following biochemical reaction network

$$
A+B \stackrel{k_{1}}{\longrightarrow} 2 B \quad B \stackrel{k_{2}}{\longrightarrow} 0 \quad A \stackrel{k_{k_{3 b}}}{\underset{k_{3 b}}{\longrightarrow}} 0 .
$$

By letting $\mathbf{c}=([A][B])^{T}$, the stoichiometric matrix and the reaction rate vector, according to the order proposed in (5) and (3), are

$$
\begin{aligned}
\mathbf{N} & =\left(\begin{array}{r|rr|r}
-1 & 0 & -1 & 1 \\
1 & -1 & 0 & 0
\end{array}\right) \\
\mathbf{v}(\mathbf{c}) & =\left(\begin{array}{llllll}
k_{1} c_{1} c_{2} & \mid & k_{2} c_{2} & k_{3 f} c_{1} & k_{3 b}
\end{array}\right)^{T} .
\end{aligned}
$$

For this system, there exist two fixed points

$$
\begin{aligned}
& \overline{\mathbf{c}}_{\mathbf{1}}=\left(\begin{array}{ll}
\frac{k_{3 b}}{k_{3 f}} & 0
\end{array}\right)^{T} \\
& \overline{\mathbf{c}}_{\mathbf{h}}=\left(\begin{array}{ll}
\frac{k_{2}}{k_{1}} & \frac{k_{3 b}}{k_{2}}-\frac{k_{3 f}}{k_{1}}
\end{array}\right)^{T}
\end{aligned}
$$

For sake of simplicity, we choose to make the Taylor expansion of the model around $\overline{\mathbf{c}}_{1}$. Hence, the Jacobian of $\mathbf{v}(\mathbf{c})$ is

$$
\mathbf{J}\left(\overline{\mathbf{c}}_{\mathbf{l}}\right)=\left(\begin{array}{cc}
0 & \frac{k_{1} k_{3 b}}{k_{3 f}} \\
0 & k_{2} \\
k_{3 f} & 0 \\
0 & 0
\end{array}\right) .
$$


In order to fully determine the PDE, we will consider that $x \in[0,1]=\Omega \subset \mathbb{R}$, with the initial concentrations (initial condition) represented by

$$
\left(\begin{array}{l}
c_{1}(0, x) \\
c_{2}(0, x)
\end{array}\right)=\left(\begin{array}{c}
\bar{c}_{1} \\
\mathcal{N}\left(x-\mu, \sigma^{2}\right)
\end{array}\right)
$$

where $\mathcal{N}(\circ)$ is a Gaussian function defined as

$$
\mathcal{N}\left(x-\mu, \sigma^{2}\right)=\frac{1}{\sqrt{2 \pi \sigma^{2}}} \exp \left(-\frac{(x-\mu)^{2}}{2 \sigma^{2}}\right) .
$$

Also, we account for no-flux boundary conditions

$$
\left.\frac{\partial \mathbf{c}(t, x)}{\partial \mathbf{n}}\right|_{x=\partial \Omega}=0
$$

We further consider that the spatial domain is long enough so as to be considered infinite.

\section{B. Reduction of Order via Analytical Solution for $y(t, x)$}

With these definitions, the linear transformation in (24) becomes

$$
\begin{aligned}
\mathbf{T} & =\left(\begin{array}{c}
\mathbf{Q} \\
\mathbf{M}
\end{array}\right)=\left(\begin{array}{rr}
1 & 1 \\
-1 & 1
\end{array}\right) \\
\mathbf{T}^{-1} & =\left(\begin{array}{ll}
\mathbf{Q}^{+} & \mathbf{M}^{+}
\end{array}\right)=\frac{1}{2}\left(\begin{array}{r|r}
1 & -1 \\
1 & 1
\end{array}\right)
\end{aligned}
$$

which imply

$$
\left(\begin{array}{l}
y(t, x) \\
z(t, x)
\end{array}\right)=\left(\begin{array}{r}
e_{1}(t, x)+e_{2}(t, x) \\
-e_{1}(t, x)+e_{2}(t, x)
\end{array}\right) .
$$

Moreover, the corresponding initial conditions in the $y, z$ variables are (recall that in this coordinates, $\overline{\mathbf{e}}=\mathbf{0}$ ):

$$
\left(\begin{array}{l}
y(0, x) \\
z(0, x)
\end{array}\right)=\left(\begin{array}{l}
\mathcal{N}\left(x-\mu, \sigma^{2}\right) \\
\mathcal{N}\left(x-\mu, \sigma^{2}\right)
\end{array}\right) .
$$

Hence Condition (22) becomes

$$
\left(\begin{array}{ll}
k_{3 f} & k_{2}
\end{array}\right)=\left(\frac{1}{2}\left[k_{3 f}+k_{2}\right] \quad \frac{1}{2}\left[k_{3 f}+k_{2}\right]\right),
$$

which is satisfied by letting $k_{3 f}=k_{2}=k$. Moreover, from Equation (23) follows $\Theta=-k$.

As stated in Section IV we can compute the spatiotemporal profile of the system by a PDE of order $r=$ $\operatorname{colrank}\left(\mathbf{N}_{\mathbf{n l}}\right)=1$. This reduced system is given by (26), which, in our case, is

$$
\begin{aligned}
\frac{\partial}{\partial t} z(t, x)= & d \nabla_{x}^{2} z+\left(\frac{k_{1} k_{3 b}}{k}-k\right) z+ \\
& \frac{k_{1} k_{3 b}}{k} y+\frac{1}{2} k_{1}\left(y^{2}-z^{2}\right) .
\end{aligned}
$$

In turn, from Lemma 4.2 and $(21), y(t, x)$ satisfies

$$
\begin{aligned}
& y(t, x)=\exp (-k t) \int_{-\infty}^{\infty} \mathcal{N}\left(x-\mu, \sigma^{2}\right) \mathcal{N}(x-\xi, 2 d t) \mathrm{d} \xi \\
& y(t, x)=\exp (-k t) \mathcal{N}\left(x-\mu, 2 d t+\sigma^{2}\right)
\end{aligned}
$$

\section{Analytical Solution \& LSD}

Now, in order to perform the simulation via the analytical solution \& LSD approach, it is necessary to compute the modes $\mathbf{w}^{\mathbf{T}}(t)$ for $y(t, x)$ and $g(y, z)$, as noted in Proposition 4.1. For $\mathbf{w}_{\mathbf{y}}^{\mathbf{T}}(t)$ we prefer the numerical computation to avoid the evaluation of a complex function in the code. In turn, from (28),

$$
\begin{aligned}
\mathbf{w}_{\mathbf{g}}^{\mathbf{T}}(t) & =\int_{\Omega} g\left(\mathbf{w}_{\mathbf{y}}^{\mathbf{T}} \boldsymbol{\phi}, \mathbf{w}_{\mathbf{z}}^{\mathbf{T}} \boldsymbol{\phi}\right) \boldsymbol{\phi}^{\boldsymbol{T}} \mathrm{d} x \\
& =\frac{k_{1}}{2} \int_{0}^{1}\left(\mathbf{w}_{\mathbf{y}}^{\mathbf{T}} \boldsymbol{\phi}\right)^{2} \boldsymbol{\phi}^{\boldsymbol{T}}-\left(\mathbf{w}_{\mathbf{z}}^{\mathbf{T}} \boldsymbol{\phi}\right)^{2} \boldsymbol{\phi}^{\boldsymbol{T}} \mathrm{d} x \\
\mathbf{w}_{\mathbf{g}}^{\mathbf{T}}(t) & =\frac{k_{1}}{2} \int_{0}^{1} \boldsymbol{\phi}^{\boldsymbol{T}}\left(\mathbf{w}_{\mathbf{y}} \mathbf{w}_{\mathbf{y}}^{\mathbf{T}}-\mathbf{w}_{\mathbf{z}} \mathbf{w}_{\mathbf{z}}^{\mathbf{T}}\right) \boldsymbol{\phi} \boldsymbol{\phi}^{\boldsymbol{T}} \mathrm{d} x .(34)
\end{aligned}
$$

The vectorised expression of the term $\phi^{T}\left(w^{T} w^{T}\right) \phi \phi^{T}$ is $\operatorname{vec}\left(\phi^{\boldsymbol{T}}\left(\mathbf{w} \mathbf{w}^{\mathbf{T}}\right) \phi \phi^{\boldsymbol{T}}\right)=\left(\phi \phi^{\boldsymbol{T}} \otimes \phi^{\boldsymbol{T}}\right) \operatorname{vec}\left(\mathbf{w} \mathbf{w}^{\mathbf{T}}\right)$

from which we note an explicit separation of the spatial and temporal dependency. Each of the terms in the matrix $\phi \phi^{\boldsymbol{T}} \otimes$ $\phi^{T}$ is a product of three elements of the basis. Their integral over the spatial domain have this simple expression

$$
\int_{0}^{1} \phi_{i} \phi_{j} \phi_{k} \mathrm{~d} x= \begin{cases}1, & i=1, j=k \forall j, k \in[1, p] \\ 0, & \text { otherwise. }\end{cases}
$$

Then we can write the Equation (34) as

$$
\begin{aligned}
\operatorname{vec}\left(\mathbf{w}_{\mathbf{g}}^{\mathbf{T}}\right)(t)= & \frac{k_{1}}{2}\left(\int_{0}^{1} \phi \boldsymbol{\phi}^{\boldsymbol{T}} \otimes \boldsymbol{\phi}^{\boldsymbol{T}} d x\right) \\
& {\left[\operatorname{vec}\left(\mathbf{w}_{\mathbf{y}}(t) \mathbf{w}_{\mathbf{y}}^{\mathbf{T}}(t)-\mathbf{w}_{\mathbf{z}}(t) \mathbf{w}_{\mathbf{z}}^{\mathbf{T}}(t)\right)\right] . }
\end{aligned}
$$

D. $L S D$

Finally, we assess the performance of the previous methods with the reduced order model obtained from (6). As stated in Section III, the weights for this PDE satisfy

$$
\begin{aligned}
\frac{d}{d t} \operatorname{vec}\left(\mathbf{W}^{\mathbf{T}}\right)= & {\left[d\left(\boldsymbol{\Lambda} \otimes \mathbf{I}_{\mathbf{2}}\right)+\left(\mathbf{I}_{\mathbf{p}} \otimes \mathbf{N}_{\mathbf{l}} \boldsymbol{\Gamma}\right)\right] \operatorname{vec}\left(\mathbf{W}^{\mathbf{T}}\right) } \\
& +\left(\mathbf{I}_{\mathbf{p}} \otimes \mathbf{N}_{\mathbf{0}}\right) \operatorname{vec}\left(\left(\mathbf{v}_{\mathbf{0}} \mathbf{0}\right)\right)+ \\
& +\left(\mathbf{I}_{\mathbf{p}} \otimes \mathbf{N}_{\mathbf{n l}}\right)\left(\int_{\Omega} \boldsymbol{\phi} \boldsymbol{\phi}^{\boldsymbol{T}} \otimes \boldsymbol{\phi}^{\boldsymbol{T}} \mathrm{d} x\right) \\
& (\mathbf{W} \otimes \mathbf{W}) \operatorname{vec}(\mathbf{P})
\end{aligned}
$$

\section{E. Implementation and Results}

The simulations were performed in a $\mathrm{PC}$ with a processor Intel Core 2 Duo at $2.66 \mathrm{GHz}$ and $3 \mathrm{~GB}$ of RAM, running Ubuntu 11.04 and Matlab 7.5.

Four different implementation were considered in order to compare the methods:

1) Numerical Implementation. We avail of the function pdepe implemented in Matlab

2) Analytical \& Numerical. Using the expression for $y(t, x)$ in (33), we simulated (32) with pdepe

3) Analytical \& LSD. We implemented (27), where $\mathbf{w}_{\mathbf{g}}^{\mathbf{T}}(t)$ have been defined in (37) and $\mathbf{w}_{\mathbf{y}}^{\mathbf{T}}(t)$ are computed numerically. In this case, we used the $O D E 15 \mathrm{~s}$ solver. 
4) LSD. The implementation of (38) was simulated by the $O D E 15 s$ solver.

A global basis for $\Omega$ with no-flux boundary conditions, which comply with (11) and (12) is given by

$$
\begin{aligned}
\phi_{i}(x) & =k_{i} \cos (2 \pi(i-1) x) \\
k_{i} & =\left\{\begin{array}{ll}
1, & i=1 \\
\sqrt{2}, & i \neq 1
\end{array} .\right.
\end{aligned}
$$

In turn, the eigenvalues are

$$
\lambda_{i}=-[2 \pi(i-1)]^{2} \quad \forall i \in[1, p] .
$$

Figure 1 shows the surfaces obtained with the four different methods. Moreover, we compared the time required for these simulation and compared them with the Numerical Implementation, by means of the expression

$$
E_{i}[\%]=100 \frac{t_{\text {numeric }}-t_{i}}{t_{i}},
$$

where $t_{\text {numeric }}$ is the time required to simulate the model with the Matlab function pdepe and $t_{i}$ stands for the time required for each other method. The results of this assessment are presented in Figure 2, which shows $E_{i}[\%]$ as a function of number of nodes in the spatial domain used for the simulation. It is important to note that this result is for $p=34$ number of eigenfunctions. As this number decreases, the LSD approach improves its performance w.r.t. the Analytical \& LSD implementation and conversely. We also note a that the value $E_{i}[\%]$ for the Analytical \& Numerical approach tends to remain constant as the number of nodes varies.

\section{CONCLUSIONS}

We presented a set of state transformations which aid to reduce the order of a class of reaction-diffusion systems so as to make their simulation quicker. The amount of the reduction depends closely to the stoichiometry of the reaction network taken into account. We note that the computational speed of the Analytical \& LSD approach out perform the rest of the methods considered, when the number of eigenfunctions used is large enough. In addition, the methods which include an analytical solution allow further dynamical study such as sensitivity and stability analysis.

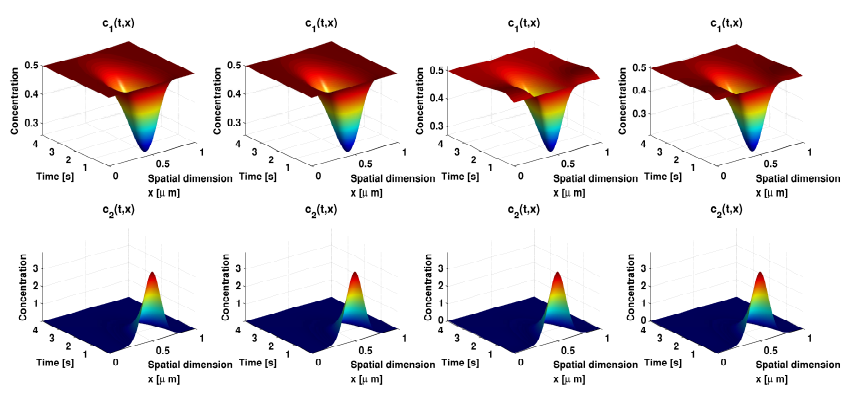

Fig. 1. Comparison of the methods for solving the PDE in the case study. From left to right this figure depicts the surface obtained by numerical, analytical/numerical, analytical/LSD, and LSD approaches, respectively.

\section{REFERENCES}

[1] N. F. Britton, Reaction-Diffusion Equations and their Applications to Biology. Academic Press, 1986.

[2] J. D. Murray, Mathematical Biology II. Springer, 3rd ed., January 2003.

[3] C. P. Fall, E. S. Marland, and J. M. Wagner, Computational Cell Biology. Berlin: Springer, February 2005.

[4] P. Christofides, Nonlinear and robust control of PDE systems: Methods and applications to transport-reaction processes. Birkhauser, 2001.

[5] C. Vilas, M. Garcia, J. Banga, and A. Alonso, "Robust feed-back control of distributed chemical reaction systems," Chemical engineering science, vol. 62, no. 11, pp. 2941-2957, 2007.

[6] R. B. Bird, W. E. Stewart, and E. N. Lightfoot, Transport Phenomena. Wiley, 2 ed., 2006.

[7] L. Sirovich, "Turbulence and the dynamics of coherent structures. Part I: Coherent structures," Quaterly of Appl. Math., vol. 45, no. 3, pp. 561-571, 1987.

[8] R. Courant and D. Hilbert, Methods of Mathematical Physics. New York, USA: Wiley, 1937

[9] A. A. Alonso and B. Ydstie, "Stabilization of distributed systems using irreversible thermodynamics," Automatica, vol. 37, no. 11, pp. 1739 $1755,2001$.

[10] A. J. Laub, Matrix Analysis For Scientists And Engineers. Philadelphia, PA, USA: Society for Industrial and Applied Mathematics, 2004.

[11] J. Jost, Partial Differential Equations. Springer, 2nd ed., January 2007.

[12] A. D. Polyanin, Handbook of Linear Partial Differential Equations for Engineers and Scientists. Chapman and Hall/CRC, 1 ed., November 2001.

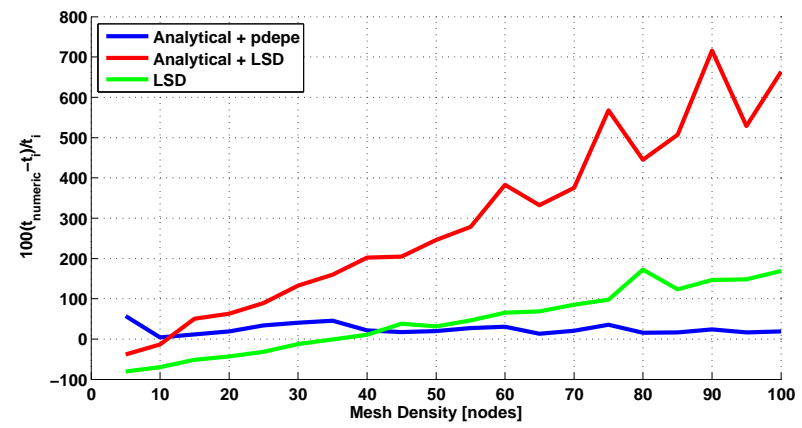

Fig. 2. CPU time assessment for the three proposed methods to solve a PDE. The parameters used for the simulation are: $p=34$, $\left\{k_{1}, k_{2}, k_{3 b}, k_{3 f}\right\}=\{1,2,2,1\}, d=0.001, \sigma^{2}=0.01, \mu=0.5$. 